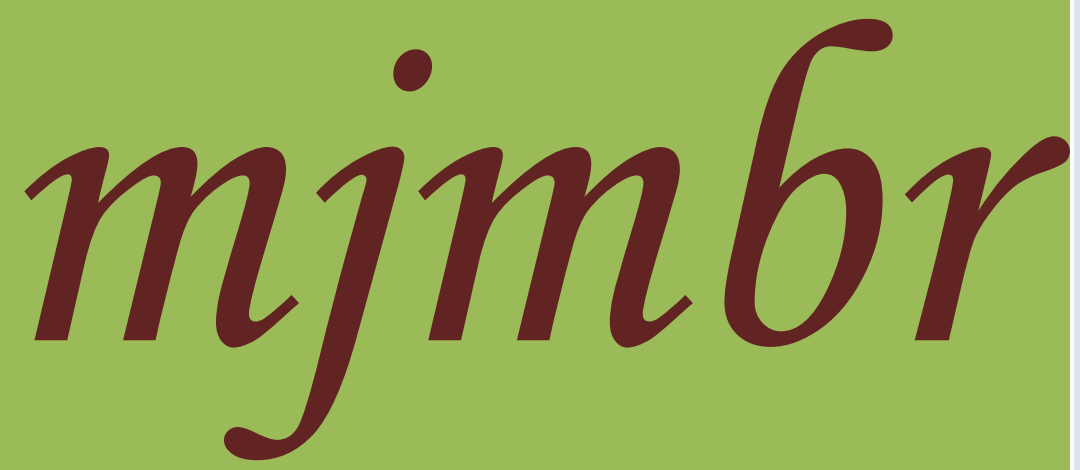

Malaysian Journal of Medical and Biological Research

www.jmbr-my.weebly.com

Vol 1, No. 1, 2014 


\title{
Core Decompression by Laser Osteoperforation: A Preliminary Report of New and Minimally Invasive Modality for the Treatment of Avascular Necrosis of the Femoral Head
}

\author{
Yakub M. Ali ${ }^{1}$, Jahangir M. Sarwar ${ }^{2}$, Manash C. Sarker ${ }^{3}$, Mohammad S. \\ Hossain $^{4}$, Muhammad N. Kayes ${ }^{5}$, Muhammed M. Rahman ${ }^{6}$, \& Hans Hainz ${ }^{7}$
}

${ }^{1}$ Director, Institute of Laser Surgery and Hospital, Dhaka-1000, Bangladesh

${ }^{2}$ Consultant, Department of Surgery, District Hospital, Sherpur-2100, Bangladesh

${ }^{3,4,6}$ Consultant Laser Surgery, Institute of Laser Surgery and Hospital, Dhaka-1000, Bangladesh

${ }^{5}$ Assistant Professor of Anesthesia \& ICU, Delta Medical College \& Hospital, Dhaka-1216, Bangladesh

${ }^{7}$ Adviser, Institute of Laser Surgery and Hospital, Dhaka-1000, Bangladesh

\begin{abstract}
Introduction: Avascular necrosis (AVN) of the femoral head is a devastating disease that often leads to the destruction of the hip. Core decompression of femoral head is the commonest of all conservative surgical treatments where an 8-10 mm cylindrical core of bone is removed from antero-lateral segment of the femoral head. But it is employed only in early stages of the disease and its results are still of controversial efficacy and most of patients ultimately require total hip arthroplasty. Laser irradiation stimulates the reparative process in the bone and marrow while local hyperthermia produced by the laser vaporizes some of necrotic areas leading to a reduction of the intraosseous pressure which is the fulcrum of AVN regardless of the cause.

Objectives: The aim of this experimental study was to evaluate the outcome of core decompression by laser osteoperforation for AVN of the femoral head.

Subjects and methods: The study was carried out at the Institute of Laser Surgery and Hospital, Dhaka, Bangladesh which included 62 hips with AVN in 40 patients. Preoperative and Postoperative Clinical assessment and Radiological staging were done using 'Harris Hip Score (HHS)' and 'Ficat and Arlet staging' respectively. Under spinal anesthesia and Fluoroscopic guidance all hips were subjected to 'Core decompression by Laser Osteo-perforation' using $970 \mathrm{~nm}$ Diode laser through 18G spinal needle via transtrochanteric and trans-capital approach.

Results: All patients were followed up at intervals of three weeks, three months, six months and then yearly (cases with minimum 3 follow ups were included in this study). According to HHS our results are as : $40.3 \%$ - excellent; $21.0 \%$ - good; $29.0 \%$ - fair; $9.7 \%$ poor. No significant side effects or complications were observed so far.

Conclusion: Core decompression by laser osteoperforation has been proven so far to be an effective, safe and minimally invasive treatment modality for AVN of the femoral head.
\end{abstract}

Keywords: AVN, Avascular necrosis, AVN of femoral head, Core decompression, Laser osteoperforation 


\section{INTRODUCTION}

Avascular necrosis (AVN) of the bone, also known as osteonecrosis, aseptic necrosis and ischemic bone necrosis, is a relatively common disease characterized by death of the cellular elements of bone and/or marrow due to the interruption of the blood supply [1]. Most individuals who develop AVN are between ages of 20 and 50 years. The hip (the femoral head) is the most commonly affected sites for clinically significant AVN.

AVN of the femoral head is a devastating disease that often leads the destruction of the hip and requires a total hip arthroplasty $[2,3]$. There are many risk factors including alcoholism, excessive use of steroids, post trauma, caisson disease (decompression sickness), vascular compression, sickle cell anemia, Gaucher's disease, and deep diving. However in some cases it is idiopathic. For non-traumatic AVN, it is often bilateral affecting only both hips, which further increases the extent of disability. Early presentation of AVN of the femoral head may be painless; however the ultimate presentation is a painful limitation of hips motion [3]. Passive movements of hip are also restricted.

Core decompression of the femoral head is the commonest of the available conservative surgical treatments. It is usually employed before the collapse and fracture of the femoral head and/or neck to delay or avoid reconstructive surgery of the affected joint. It is generally carried out to preserve the function and the structure of the hip as well as to relieve pain associated with AVN. Core decompression entails repair of the necrotic site by coring, followed by filling the cored area with a bone graft, but this is optional. A lateral trochanteric approach is used in this procedure which involves an 8-mm to $10-\mathrm{mm}$ cylindrical core of bone being removed from the anterolateral segment of the femoral head, to create an open cylindrical channel. This open channel serves to relieve pressure. The open channel may then be filled with either a vascularized or a non-vascularized bone graft. The former is used to aid in growth of vascular cellular tissue in the necrotic area thus enhancing revascularization, which may arrest the progression of the necrosis. The latter is used to provide structural stability to the hip during the healing process.

The result of core decompression of the femoral head is still of controversial efficacy. Most of patients ultimately require total hip arthroplasty. Even the result of total hip replacement (THR) and hemiarthroplasty has generally been poorer in patients who have AVN. The high cost of replacement procedures are far beyond the reach of most patients in Bangladesh, whose population hails mostly from a poor/middle socioeconomic class. As a result there is a constant search for newer techniques. Laser osteoperforation has been proved to be effective for treatment of inflammatory and destructive bone diseases in both experimental [4] and clinical settings [5]. Laser irradiation stimulates the reparative processes in bone and marrow while local hyperthermia produced by the laser vaporizes some of necrotic areas reducing the raised intraosseous pressure which is the fulcrum of AVN whatever the cause is.

The general objective of the presented experimental study was to assess the scope of core decompression by laser osteoperforation as a new and minimally invasive modality for the treatment of AVN of the femoral head.

The specific objective was to assess the safety and success of laser osteoperforation for the treatment of:

a) Early/pre-collapse AVN of femoral head (stage I or II i.e. before X-ray changes are evident) and thereby not only cure the disease but also conserve the structural and functional integrity of the affected hip. 
b) Late/post-collapse AVN of femoral head (stage III or higher; when X-ray changes have occurred) and thereby avoids and/or delay the need for a THR or hemiarthroplasty by halting the disease process and making the patient pain free, particularly for those who cannot afford the high cost replacement procedures or are unfit for such a procedure.

\section{SuBJECTS AND MethodS}

At a glance

- Study design: Non-randomized controlled trial (NRCT)

- Place of study: Institute of Laser Surgery and Hospital, Dhaka, Bangladesh.

- Period of study: July 2009 to March 18, 2013 (with a minimum of three follow-ups as per schedule).

- Study population: A total of 40 patients presenting 62 hips with AVN of the femoral head were included in the study regardless of the cause and gender.

- Informed written consent was obtained from all subjects in a prescribe form.

- Ethical clearance was obtained from the appropriate authority i.e BMRC (Bangladesh Medical Research Council), Dhaka, Bangladesh.

\section{Criteria for patient selection}

Participants of the study were recruited from the patients with AVN of the femoral head who had attended the Institute of Laser Surgery and Hospital, Dhaka, Bangladesh.

The inclusion criteria were:

- $\quad$ Patients with AVN of the femoral head diagnosed in the hospital.

- Diagnosis was confirmed by clinical findings, radiographs and magnetic resonance imaging (MRI).

The exclusion criteria were:

- Patients not willing to participate.

- Patients with acute emergency condition.

- Patients with psychological disorders.

- Patients with concurrent fractures.

- Patients with systemic disease, such as uncontrolled diabetes, chronic infection etc.

Pre and post operative evaluation was conducted with:

- $\quad$ Harris Hip Score (HHS) [6, 7].

- Antero-posterior and lateral radiographs of the hip joint.(For Ficat and Arlet staging)[8]

- $\quad$ MRI of the hip joint.(Selective cases)

\section{Equipment/material used:}

- Fluoroscopy unit (Siemens, Germany)

- Fluoroscopy compatible operating table (Siemens,Germany)

- Surgical laser (970 nm diode laser, type: LAHTA MILON; Milon Group, StPetersburg, Russia)

- Spinal needle 18G, 88 mm (Spinocan; B Braun,Germany) or 16G, 90 mm (made in China)

- Sterile WF 400/440/465P Poliimid with SMA-905 connector optical fiber for delivering laser energy (made in Russia) 


\section{Technique}

Under spinal anesthesia patients were positioned supine on the operating table. After antiseptic painting and draping one to three $18 \mathrm{G}$ spinal needles were inserted at and just below the greater trochanter aiming towards the femoral head under fluoroscopic guidance to carry the quartz monofiber $(0.4 \mathrm{~mm}$ diameter) from skin to the bone; then trans-trochenteric (Figure $1 \mathrm{a})$ laser osteoperforations were done extending from the bony cortex to the center of the necrotic lesion in the head using $970 \mathrm{~nm}$ diode laser. These perforations are intended to make fine tunnels from the healthy trochenteric and neck region of the femur to the necrotic region of the head. Their calibers are narrow but sufficient enough to reduce intra osseous pressure and allow angiogenesis and other laser induced reparative processes through them. Again three to five trans-capital (perforating anterior cortex of the head) (Figure 1b) laser osteoperforations were made using $18 \mathrm{G}$ spinal needles according to the size of the lesion. These perforations produce tunnels through which most of the necrotic tissue boils off/ evaporate and thus decompressing the head yet further. The fineness of the entry wound directly over the head did not affect the structural or functional integrity of the head very much. Laser was applied at 30 watts in continuous mode for 3 to 5 minutes for each trans-trochenteric perforation and at 16-20 watt in 10 milli-second pulse mode for 1 to 3 minutes for each trans-capital perforation. Post operatively all patients were kept in absolute bed rest for three weeks to prevent iatrogenic fracture / collapse of the head and/or the neck. Then progressively patients were allowed for non weight bearing to all ranges of weight bearing movements.

All patients were followed-up at intervals of three weeks, three months, six months and then yearly. Cases with minimum 3 follow ups were included in this study.

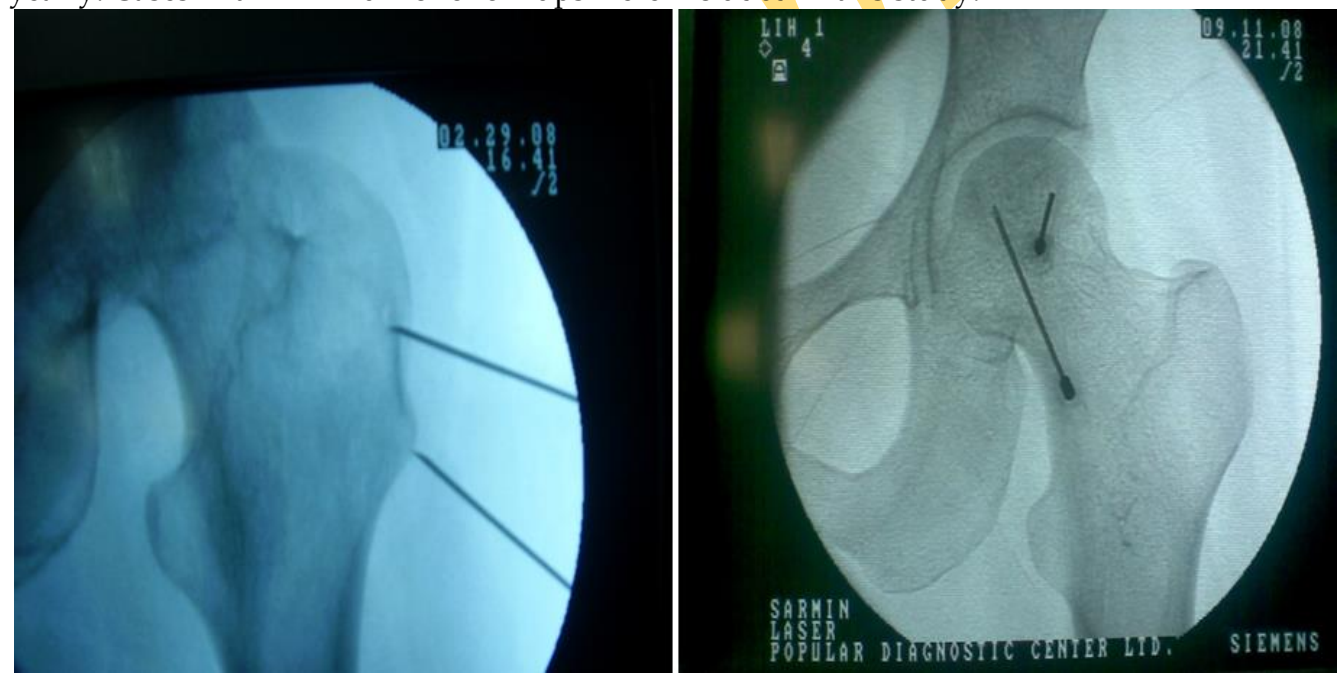

Figure 1: Placement of needles under Fluoroscopic guidance for (a) trans-trochanteric and (b) trans-capital osteoperforation.

\section{Outcome measurement}

Antero-posterior and lateral radiographs were taken immediately before surgery and at all follow-up examinations. Clinical hip evaluation was carried out according to the Harris hip scoring (HHS) system where several clinical parameters (pain, distance walked, activities, use of transport, use of supports during walking, limp, climbing stairs, sitting, range of movements) are evaluated by numerical scoring. A score between 90-100 refers to excellent result, where as 80-89 - good, 70-79 - fair and $<70$ stands for poor result. 


\section{RESULTS}

A total of 40 patients presenting 62 hips with AVN were included in this study, of which 28 were male and 12 were female. The age ranged between 21 and 90 years (mean 37.4 years) (Table 1). In 22 patients both hips were affected, in 10 patients it was the right hip and in 8 patients it was the left hip that was diagnosed with AVN of the femoral head. The main causes of AVN in this study were $55 \%$ drug (steroid) induced, $25 \%$ idiopathic and $20 \%$ post-traumatic. Due to its delay at presentation and diagnosis most of the hips (37 out of 62) were found in Ficat Stage III and IV, while 15 and 10 hips were at stage I and II at presentation respectively (Table 2).

Table 1 Age distribution of patients

\begin{tabular}{ccc}
\hline Age range (years) & Number of patients & $\mathbf{\%}$ \\
\hline $20-30$ & 14 & $35 \%$ \\
$31-40$ & 14 & $35 \%$ \\
$41-50$ & 5 & $12.5 \%$ \\
$51-60$ & 5 & $12.5 \%$ \\
$61-70$ & 1 & $2.5 \%$ \\
$>71$ & 1 & $2.5 \%$ \\
Total & 40 & 100 \\
\hline
\end{tabular}

Table 2 Distribution of involved hips according to radiological stage (Ficat and Arlet, 1980)

\begin{tabular}{ccc}
\hline Ficat and Arlet stage & Number of hips & $\mathbf{\%}$ \\
\hline Stage I & 15 & $24.19 \%$ \\
Stage II & 10 & $16.13 \%$ \\
Stage III & 17 & $27.47 \%$ \\
Stage IV & 20 & $32.26 \%$ \\
Total & 62 & 100 \\
\hline
\end{tabular}

Typically pain was reduced in most patients and their day to day activities were improved and there was varying degree of improvement in the range of hip movements as well. These were evaluated with HHS. There had been significant radiological improvement (down staging) in stage III and a few stage IV hips.

Pre-operative HHS had an average value of 31.4 whereas postoperative HHS had an average value of 82.4 .

After three follow ups (i.e after 10 to 12 months from date of operation), according to HHS our results are as follows: $40.3 \%$ - excellent; $21.0 \%$ - good; $29.0 \%$ - fair; $9.7 \%$ - poor (Table 3).

Table 3 Outcome according to HHS. after a minimum of 3 follow ups (at ten months from operation) onwards.

\begin{tabular}{lll}
\hline Outcome & Number & $\mathbf{\%}$ \\
\hline Excellent & $\mathbf{2 5}$ & 40.3 \\
Good & $\mathbf{1 3}$ & 21.0 \\
Fair & $\mathbf{1 8}$ & 29.0 \\
Poor & $\mathbf{6}$ & 9.7 \\
Total & & 100.0 \\
\hline
\end{tabular}

According to Ficat stage we found excellent result $80 \%$ in stage I, $60 \%$ in stage II, $23.53 \%$ in stage III and $15 \%$ in stage IV (Table-4). 
Malaysian Journal of Medical and Biological Research, Volume 1, No 1 (2014)

Table 4 Outcome according to HHS. After a minimum of 3 follow ups (at ten months from operation) onwards.

\begin{tabular}{cccccc}
\hline Stage & $\begin{array}{c}\text { Outcome }> \\
\text { No. of Patients }\end{array}$ & Excellent & Good & Fair & Poor \\
\hline Stage I & 15 & $12(80 \%)$ & $2(13.33 \%)$ & $1(6.67 \%)$ & 0 \\
\hline Stage II & 10 & $6(60 \%)$ & $2(20 \%)$ & $2(20 \%)$ & 0 \\
\hline Stage III & 17 & $4(23.53 \%)$ & $5(29.41 \%)$ & $7(4.18 \%)$ & $1(5.88 \%)$ \\
\hline Stage IV & 20 & $3(15 \%)$ & $4(20 \%)$ & $8(40 \%)$ & $5(25 \%)$ \\
\hline Total (n) & 62 & $25(40.3 \%)$ & $13(21.0 \%)$ & $18(29.0 \%)$ & $6(9.7 \%)$ \\
\hline
\end{tabular}

Figure 2a, 2b (Preoperative) and Figure 3a, 3b (Postoperative) show significant clinical improvement in the same patient, whose pre-operative and postoperative HHS was 37 and 90 respectively.
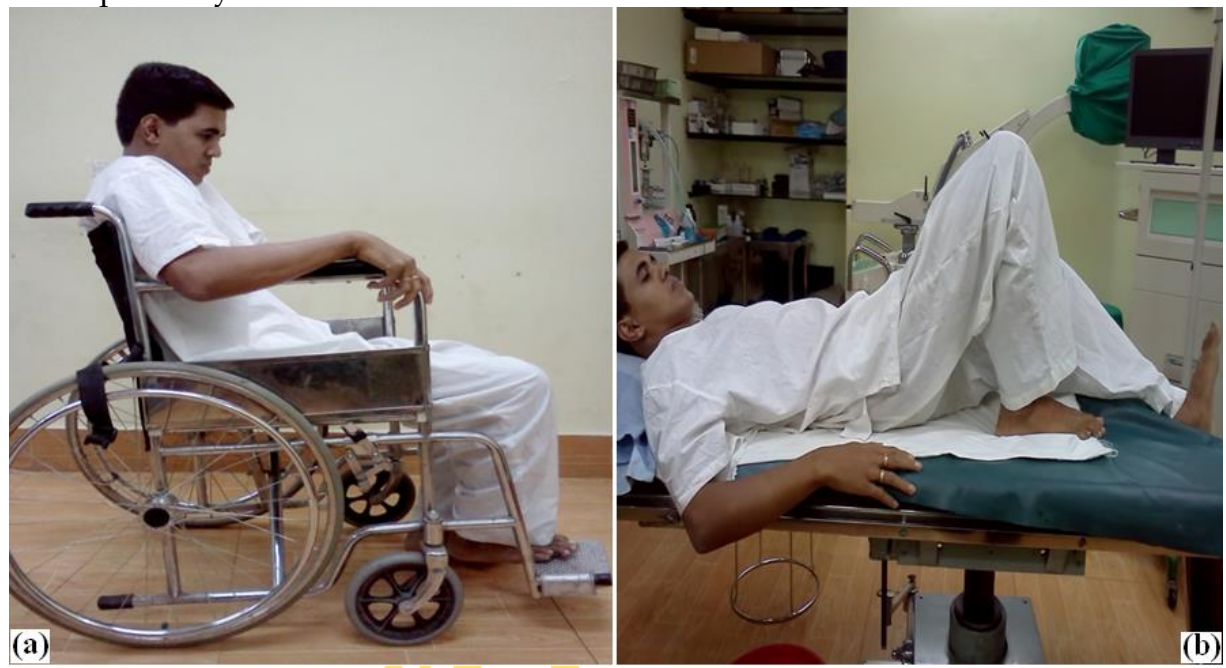

Figure 2: Pre-operative picture of a 28-years old male patient (a) unable to walk, bound to wheel chair due to limitation of hip movement and (b) Fixed flexion at right hip, buttock does not touch the bed.
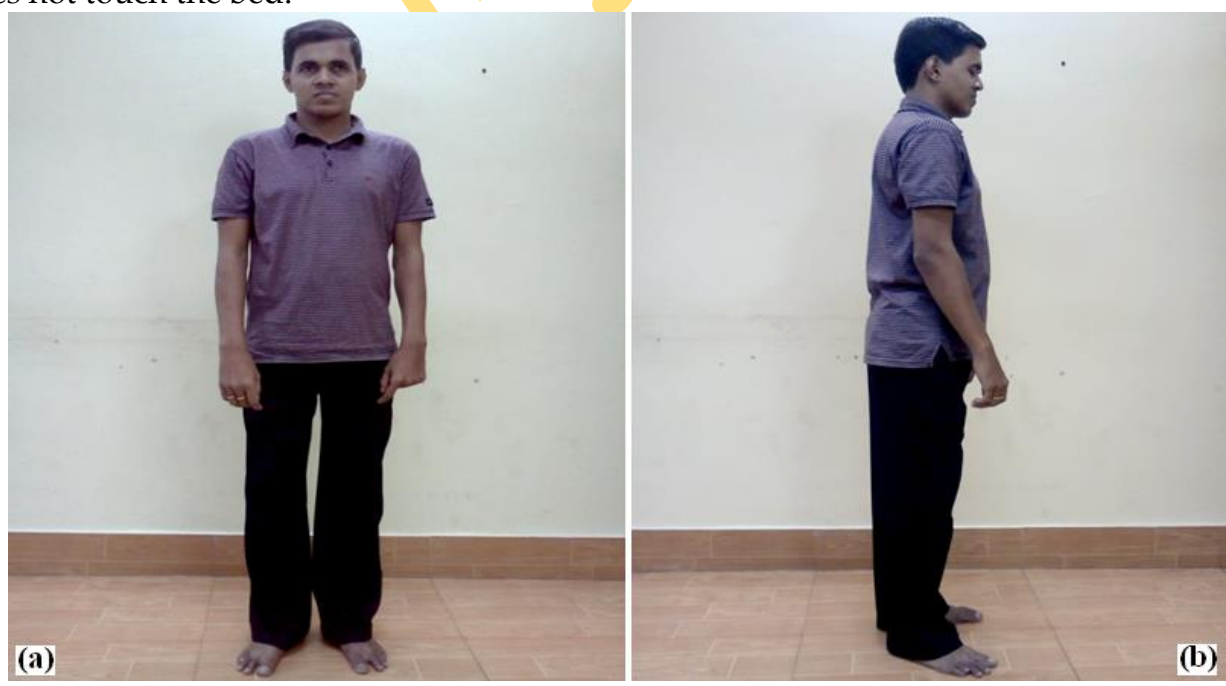
Figure 3: Postoperative picture of the same patient as in figure 2, (a) front view - patient can stand and walk without support, no apparent limb length discrepancy (b) Lateral view - no flexion deformity

Figure 4a (Preoperative) and 4b (Postoperative) X-ray pelvis A/P views of a 38 years old patient illustrate radiological down-staging from stage IV to stage II. His pre-operative and postoperative HHS was 15 and 85 respectively.
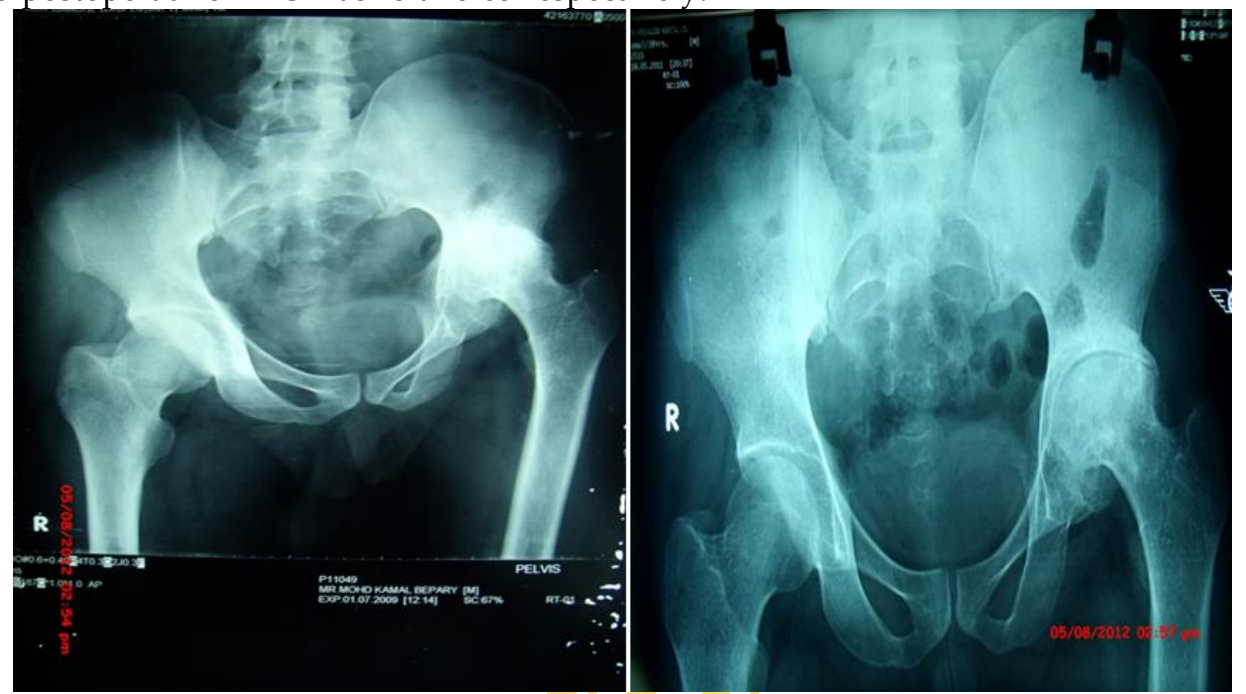

Figure 4a: Pre-operative $\mathrm{X}$-ray Pelvis A/P view of a 38 years old male patient showing-

1. a. Low density areas of diffuse osteonecrosis with peripheral osteosclerotic changes are seen at left sided femoral head and acetabulum associated with subchondral fracture.

b. Hypodense areas with osteosclerotic changes are also noticed at head and acetabulum with secondary narrowing of joint space are noticed, joint space is mostly obliterated.

c. Osteolytic change with disfiguration / mild flattening of the head of the femur is also evident.

2. Striated soft tissue areas are seen around the left hip joint; possibly the pannus tissue formation as a sequel of chronic arthritis.

3. Mild osteoporotic changes are also noticed at neck, trochenter and meta-diaphysis of the left femur.

Figure 4b: Post-operative $\mathrm{X}$-ray Pelvis $\mathrm{A} / \mathrm{P}$ view of the same patient as in Figure 4a. showing-

1. Hypodense areas with osteoslerotic changes are noticed at head \& acetabulum with secondary narrowing of joint space are noticed. Osteolytic change with mild flattening of head of the femur also evident.

2. Diffuse osteonecrosis are reduced, joint space clearly visualized with mostly smooth margins of opposing surfaces of acetabulum \& head of the left femur; those are obscured in earlier X-ray on 01.07.2009.

3. Osteoporosis seen at the neck, trochanter \& meta-diaphysis of the left femur is improved with almost normal bone density since earlier X-ray on 01.07.2009. 
We observed complications only in 2 (two) hips (3.23\%)

1. One patient developed a Puncture site infection, which was treated with appropriate antibiotic.

2. In another case there was breakage and impaction of the needle due to early lasing within the needle. The impacted portion of the needle was extracted through a small incision along the tract.

But following events also need to be kept in mind -

1. Hemarthrosis of hip joint

2. Fragmentation of femoral head

3. Fracture neck of femur

4. Soft tissue injury due to misdirected needle puncture.

5. Injury to Femoral vessels and nerve.

\section{DISCUSSION}

Bangladesh is a developing country and most of the patients with AVN hail from a low socioeconomic condition. Due to its vague presentation and absence of radiological findings in the early stages (stage I and II) of the disease most patients are wrongly diagnosed and are maltreated in these stages. Most of them could not afford an MRI in these stages. As a result, they end up in stages III and IV before proper diagnosis or treatment could be offered, which is reflected in this study even, where 37 out of 62 hips were in stages III and IV.

Core decompression is usually recommended in stages I and II. In Bangladesh THR is done only in limited centers and its cost is beyond reach of common population. So we carried out core decompression by laser osteoperforation in all stages to evaluate the clinical outcome of patients.

In this study average age of patients was 37.4 years where as Won S S et al. 2006,[10] reported mean age 36.1 years in his study which is similar with the present study .

We observed significant clinical improvements in most of the early stage diseases and some of the late cases. Even some late stage cases showed significant radiological down staging.

In 1996, Mont M A et al.[11] reported 84\% clinical success(HHS > 80) in Ficat stage I, 65\% in stage II and $47 \%$ in stage III where as in present study we found $93 \%$ clinical success in Ficat stage I, $80 \%$ in stage II, $52.94 \%$ in stage III which reflects better outcome .

In 1955, Koo et al. [9] Reported only symptomatic relief after core decompression in a small number of patients with AVN of the hip, but no effect in preventing femoral head collapse when compared with non-operative treatment. The treatment of AVN of the femoral head, particularly in young patients, remains controversial as regards conservative procedures and arthroplasty.

In this study, the average age $(<40)$ of patients was not suitable for THR whereas laser core decompression improved the clinical outcome making it possible to delay arthroplasty. None of the patients in this study have to have arthroplasty as yet.

\section{CONCLUSION}

According to this study, core decompression by laser osteoperforation has been proven so far to be an effective treatment modality for AVN of the femoral head. It is safe, minimally invasive, effective at all stages of the disease and can be repeated if needed. However, further study is required before final conclusions can be drawn. 


\section{ACKNOWLEDGEMENTS}

We are very much grateful to all our patients for their consent to be the subject of this innovative study.

We are also grateful to Dr. Abul Mokarim (Ex Major), Chief Consultant, Ibn Sina Medical Imaging Center for his valuable radiological comments.

\section{REFERENCES}

Ficat RP, Arlet J. Ischemia and Necroses of Bone (edited and adapted by DS Hungerford), Williams \& Wilkins, Baltimore,1980. [8]

Harris WH. Traumatic arthritis of the hip after dislocation and acetabular fractures: treatment by mold arthroplasty. An end-result study using a new method of result evaluation. J Bone Joint Surg Am. 1969 Jun;51(4):737-55. [6]

Koo KH, Kim R, Ko GH, Song HR, Jeong ST, Cho SH. Preventing collapse in early osteonecrosis of the femoral head. A randomised clinical trial of core decompression. J Bone Joint Surg Br 1995;77(6):870-4. [9]

Marchetti P, Binazzi R, Vaccari V, Girolami M, Morici F, Impallomeni C, Commessatti M, Silvello L. Long term result with cementless Fitek or Fitmore cups. J Arthroplasty. 2005 sep;20(6):730-7. [7]

Mont MA, Carbone JJ, Fairbank AC. Core decompression versus nonoperative management for osteonecrosis of the hip. Clinical orthopaedics and related research 1996: vol 324, pp169-178. [11]

Mont MA, Hungerford DS. Non-traumatic avascular necrosis of the femoral head. J Bone Joint Surg Am 1995;77(3):459-74. [2]

Mont MA, Jones LC, Hungerford DS. Nontraumatic osteonecrosis of the femoral head: ten years later. J Bone Joint Surg Am 2006;88(5):1117-32. Erratum in: J Bone Joint Surg Am 2006;88(7):1602. Dosage error in article text. [3]

Privalov VA, Krochek IV, Abushkin IA, Shumilin II, Lappa AV. Laser osteoperforation for treatment of inflammatory and destructive bone diseases. Proc SPIE 2009;7373:73731T. doi:10.1117/12.831939. [5]

Privalov VA, Svetlakov AL, Kuchakovsky OS, Lappa AV, Kazakov AA, Kozel AI. Hyperthermal effect of laser osteoperforation in a treatment of experimental acute purulent osteomyelitis. Proc SPIE 1999;3565:72-9. doi:10.1117/12.335787. [4]

Tofferi JK, Gilliland W. Avascular necrosis. Medscape 2012. http:/ / emedicine.medscape.com/article/333364-overview [Accessed on September 23, 2013]. [1]

Won Seok Song, Jeong Joon Yoo, Young-Min Kim, Hee Joong Kim. Results of multiple drilling compared with those of conventional methods of core decompression. Clinical orthopaedics and related research 2006: vol 454, pp139-146. [10]

\section{mjmbr !!!}

"Speedy publication service, Online archives, Paperless, web-based peer review system, Open access policy, Indexing in world known citation databases, Global circulation, Broad international readership and authorship, Online submission system, Minimum publication charge" 